\title{
WestVirginiaUniversity
}

THE RESEARCH REPOSITORY @ WVU

Graduate Theses, Dissertations, and Problem Reports

2003

\section{Dapsone activation of CYP2C9 allelic variants}

\author{
Matthew Aaron Hummel \\ West Virginia University
}

Follow this and additional works at: https://researchrepository.wvu.edu/etd

\section{Recommended Citation}

Hummel, Matthew Aaron, "Dapsone activation of CYP2C9 allelic variants" (2003). Graduate Theses, Dissertations, and Problem Reports. 1737.

https://researchrepository.wvu.edu/etd/1737

This Thesis is protected by copyright and/or related rights. It has been brought to you by the The Research Repository @ WVU with permission from the rights-holder(s). You are free to use this Thesis in any way that is permitted by the copyright and related rights legislation that applies to your use. For other uses you must obtain permission from the rights-holder(s) directly, unless additional rights are indicated by a Creative Commons license in the record and/ or on the work itself. This Thesis has been accepted for inclusion in WVU Graduate Theses, Dissertations, and Problem Reports collection by an authorized administrator of The Research Repository @ WVU. For more information, please contact researchrepository@mail.wvu.edu. 


\title{
DAPSONE ACTIVATION OF CYP2C9 ALLELIC VARIANTS
}

\section{Matthew Aaron Hummel}

\author{
A THESIS \\ Submitted to \\ The School of Pharmacy \\ at \\ West Virginia University
}

In partial fulfillment of the requirements

for the degree of

MASTER OF SCIENCE

In

Basic Pharmaceutical Sciences

Timothy S. Tracy, Ph.D., Chair

Robert L. Haining, Ph. D.

Reginald F. Frye, Ph. D.

Department of Basic Pharmaceutical Sciences

Morgantown, West Virginia

2003

Keywords: CYP2C9.1, CYP2C9.2, CYP2C9.3, CYP2C9.5, flurbiprofen, naproxen, dapsone, activation 


\section{ABSTRACT \\ DAPSONE ACTIVATION OF CYP2C9 ALLELIC VARIANTS \\ Matthew Aaron Hummel}

Studies have shown that CYP2C9.1 mediated metabolism of flurbiprofen and naproxen is activated by dapsone. However, dapsone activation has not been studied in the allelic variant forms of CYP2C9. Six concentrations of flurbiprofen $(2-300 \mu \mathrm{M})$ or naproxen (10-1800 $\mu \mathrm{M})$ were co-incubated with six concentrations of dapsone $(0-100 \mu \mathrm{M})$ and with reconstituted, purified CYP2C9.1, CYP2C9.2 (R144C), CYP2C9.3 (I359L), or CYP2C9.5 (D360E), in order to assess degrees of activation. Dapsone increased the efficicency (Vmax/Km) of flurbiprofen 4'-hydroxylation $690 \%, 3029 \%, 4600 \%$, and 2100\% by CYP2C9.1, CYP2C9.2, CYP2C9.3, and CYP2C9.5, respectively. Dapsone increased the efficiency of naproxen demethylation $591 \%, 1400 \%, 1150 \%$, and $2100 \%$, by CYP2C9.1, CYP2C9.2, CYP2C9.3, and CYP2C9.5, respectively. Also, dapsone normalized naproxen's kinetic profile from biphasic (CYP2C9.1 and CYP2C9.2) or linear (CYP2C9.3 and CYP2C9.5) to hyperbolic for all variant forms. The amino acid substitutions of CYP2C9 allelic variants appear to affect the degree of dapsone activation. 


\section{ACKNOWLEDGMENTS}

I would first like to thank my advisor, Dr. Tim Tracy, for his advice, confidence in me, and discussions about science and other topics. He has truly been a tremendous mentor and help to me in this process. I would also like to thank Dr. Rob Haining and Dr. Reggie Frye for agreeing to serve on my committee. Also, thanks go out to the entire Basic Pharmaceutical Sciences Department, faculty, staff, and students, especially those in Dr. Tracy's lab, for all of their assistance in this endeavor. Lastly, I would also like to thank my family and friends for all of their love, encouragement, and support. 


\section{TABLE OF CONTENTS}

DAPSONE ACTIVATION OF CYP2C9 ALLELIC VARIANTS ........................... i

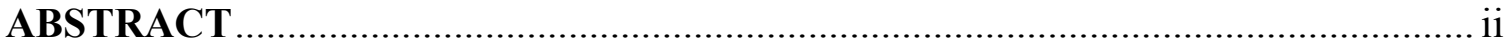

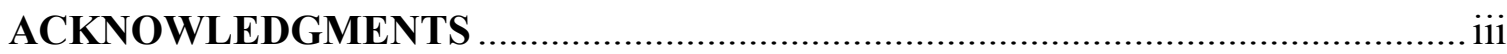

TABLE OF CONTENTS …........................................................................... iv

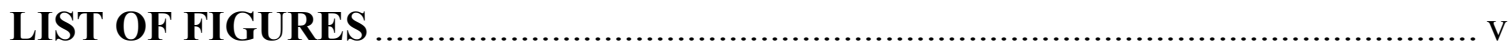

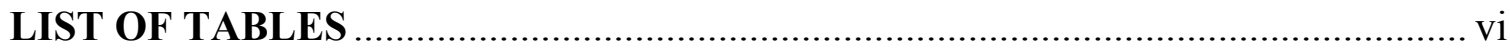

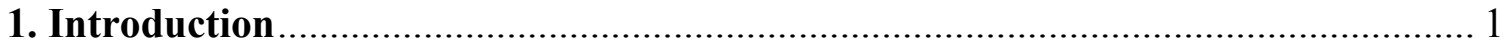

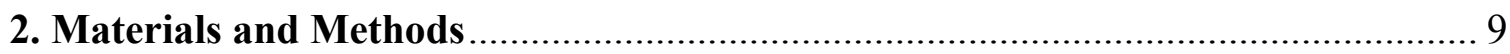

3. Dapsone Activation of CYP2C9 Allelic Variants ............................................. 15

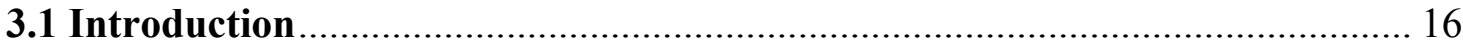

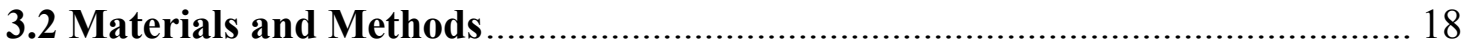

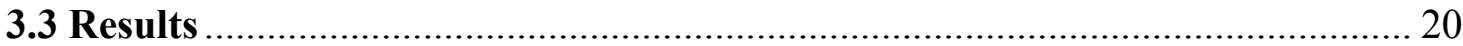

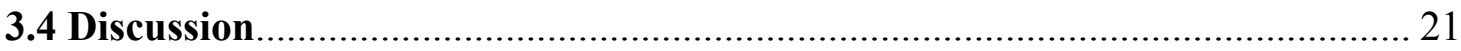

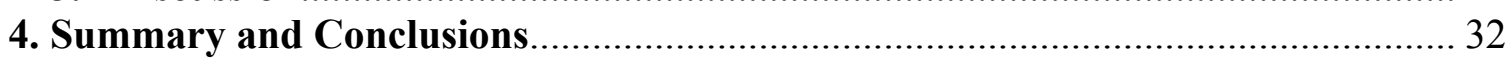

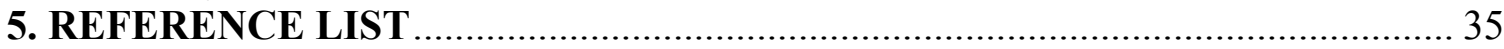




\section{LIST OF FIGURES}

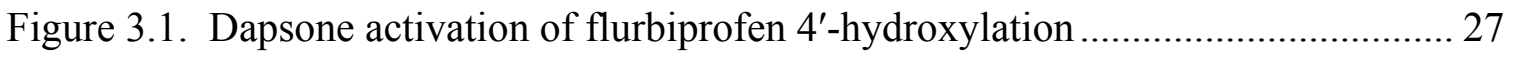

Figure 3.2. Dapsone activation of naproxen demethylation ...................................... 28 


\section{LIST OF TABLES}

Table 3.1. Kinetic Parameter Estimates for Flurbiprofen 4'-hydroxylation. .................. 29

Table 3.2. Kinetic Parameter Estimates for Naproxen Demethylation........................... 30

Table 3.3. Percent increase in efficiency $(\mathrm{Vmax} / \mathrm{Km})$ of metabolism due to dapsone

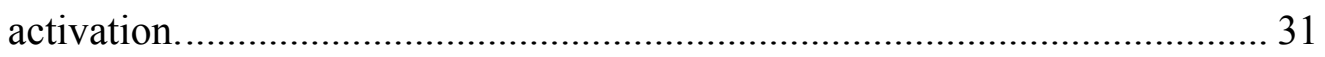




\title{
CHAPTER I
}

\author{
1. Introduction
}


Cytochrome P450's are a superfamily of mono-oxygenase enzymes that are responsible for the detoxification of exogenous as well as endogenous compounds, are found mainly in the liver, but are also present in other locations in the body, such as, the gut, skin, and lungs (Guengerich, 1995). Of the CYP2C subfamily, CYP2C9 is the major isoform comprising roughly $20 \%$ of total liver P450 content (Guengerich, 1995). As with other P450's, CYP2C9 metabolizes a range of substrates, including S-warfarin, phenytoin, tolbutamide, and a number NSAIDS, including flurbiprofen, naproxen, and piroxicam (Leemann et al., 1993;Rettie et al., 1992;Rodrigues et al., 1996;Tracy et al., 1996; Veronese et al., 1991b;Zhao et al., 1992). To date, six allelic forms, including the most common or wild type, have been discovered. Each of the five variants exhibits altered substrate metabolism, resulting in a decrease in the maximal rate of turnover and/or binding capacity. These reductions in substrate turnover are particularly important when dealing with low therapeutic index drugs such as warfarin and phenytoin (Furuya et al., 1995; Hashimoto et al., 1996;Rettie et al., 1994). The allelic variants differ from the wild type by only one amino acid residue, which occurs as a result of a single nucleotide polymorphism.

CYP2C9.1 is the wild type form, and so, is found most often in the population. The homozygous genotype, or $* 1 * l$, is found in roughly $66 \%$ of the Caucasian population (Lee et al., 2002). This genotype is even more prevalent in the Asian and African-American population, being expressed in more than $90 \%$ of the population (Lee et al., 2002).

The CYP2C9.2 variant has a cysteine residue in place of an arginine residue at amino acid position 144 due to the substitution of a cytosine for a thymine at nucleotide 
430 (Bhasker et al., 1997). Approximately $20 \%$ of the Caucasian population are heterozygous, $* 1 * 2$, with about $1 \%$ coding for the homozygous $* 2 * 2$, and about $1 \%$ coding for $* 2 * 3$ genotype (Lee et al., 2002). So far, there have been no occurrences of the $* 2$ allele discovered in Asian individuals (Lee et al., 2002). About $5 \%$ of the African-American population have been genotyped with a $* 2$ allele (Dickmann et al., 2001). Although CYP2C9.2 has been shown to reduce the metabolism of some substrates, amino acid position 144 is not thought to be in the active site of the enzyme. Crespi and Miller have implicated this substitution in reducing the interaction of the P450 with NADPH reductase, an essential protein necessary for the electron flow that is required for the oxidation of substrates (Crespi and Miller, 1997). At $\mathrm{pH} 7.4$, this effect may be due to the charged arginine residue making an electrostatic contact with the reductase, whereas the cysteine side chain is polar, but not charged. A number of in-vitro studies have been performed to examine the effects of this amino acid substitution. For example, Yamazaki and colleagues demonstrated that human liver microsomes taken from individuals genotyped heterozygous $* 1 * 2$ had an intrinsic clearance for S-warfarin, diclofenac and flurbiprofen that was $41 \%, 65 \%$, and $36 \%$ of the wild type activity, respectively, with the difference being accounted for mainly by the differences in Vmax (Yamazaki et al., 1998). The CYP2C9.2 variant has also been studied for its effects on in-vivo metabolism of CYP2C9 substrates. A study conducted by Aithal et. al., showed evidence of lower warfarin dose requirements for patients genotyped with a $C Y P 2 C 9 * 2$ allele than those with the wild type form (Aithal et al., 1999).

The CYP2C9.3 variant has probably the slightest amino acid difference, having a leucine residue in place of an isoleucine residue at amino acid position 359 as a result of 
cytosine being in the place of adenine at nucleotide 1075 (Bhasker et al., 1997). In the Caucasian population, about $10 \%$ of individuals are heterozygous, $* 1 * 3$, while about $0.5 \%$ are homozygous, $* 3 * 3$ (Lee et al., 2002). $2.5 \%$ of the African-American population was genotyped as heterozygous $* 1 * 3$ (Dickmann et al., 2001). This variant has yet to be observed in Asian individuals (Lee et al., 2002). Amino acid position 359 is thought to be in substrate recognition site (SRS) 5 according to Gotoh and may have a role in substrate binding (Gotoh, 1992). The change from isoleucine to leucine is not a major one, since they are isoforms. The only difference between the two amino acids is the position of the methyl group on the side chain. The change may alter the size of the binding pocket and cause differences in steric hinderance compared to the wild type protein or affect protein folding. This variant has been shown to affect the metabolism of CYP2C9 substrates in-vitro as well as in-vivo. Many studies have been undertaken in order to further characterize the effects of the I359L substitution on metabolism. For example, Takanashi et. al., have shown by using yeast expressed CYP2C9.3 and seven substrates, that this variant form reduces the intrinsic clearance of substrates by reducing Vmax as well as increasing Km albeit to different degrees, depending on the substrate (Takanashi et al., 2000). In order to demonstrate in-vivo consequences of the CYP2C9.3 variant, Steward et. al., described a homozygous $* 3 * 3$ patient that was receiving a warfarin dose of $0.5 \mathrm{mg} /$ day, which is substantially lower than the control patients' dose of 4-8 mg/day (Steward et al., 1997).

The CYP2C9.4 variant has a threonine residue in place of the isoleucine residue at position 359 (Imai et al., 2000). The change is a result of a substitution of thymine for cytosine at nucleotide 1076 (Imai et al., 2000). As with the CYP2C9.3 variant, this 
amino acid change is thought to be in the active site (Gotoh, 1992). The fact that the hydrophilic threonine residue is substituted for the hydrophobic isoleucine residue may play a role in the altered metabolism of substrates. The I359T mutation is very rare and was found in Japanese epileptic patients with decreased phenytoin clearance (Imai et al., 2000). In another study, this variant was found in less than $2 \%$ of Japanese subjects, and the in-vitro intrinsic clearance of CYP2C9.4 was found to be about 5 fold lower than CYP2C9.1 for diclofenac oxidation (Ieiri et al., 2000). The effect on Vmax/Km was mainly due to a lowered Vmax, with little effect on Km (Ieiri et al., 2000). The in-vivo effects of CYP2C9.4 have not yet been well characterized, but were found to be similar to CYP2C9.3 in one patient for a single dose of phenytoin (Ieiri et al., 2000).

The CYP2C9.5 variant has a glutamate residue in place of an aspartate residue at position 360 as a result of a cytosine to guanine change at nucleotide 1080 (Dickmann et al., 2001). Approximately 3\% of the African-American population are heterozygous, $* 1 * 5$, for this variant with no subjects being identified as homozygous, $* 5 * 5$ (Dickmann et al., 2001). The CYP2C $9 * 5$ allele was also recently found in $1 \%$ of a black African population from Tanzania (Yasar et al., 2002). So far, no Caucasian or Asian subjects have been genotyped with a *5 allele. Again, the site of the amino acid change is in SRS 5 , so this change is presumed to cause an alteration of the metabolism of CYP2C9 substrates (Gotoh, 1992). As with CYP2C9.3, the amino acid change is a minor one. The glutamate residue of the mutant has one extra carbon in the side chain compared to the aspartate in the wild type form, possibly making the active site smaller and causing greater steric hinderance or affecting protein folding. Dickmann et al. have shown that the $\mathrm{Vmax} / \mathrm{Km}$ for lauric acid, diclofenac, and S-warfarin metabolism is $9 \%, 18 \%$, and $8 \%$ 
compared to wild type, respectively (Dickmann et al., 2001). The effect on Vmax/Km was mainly due to an increase in Km with little effect on Vmax for these substrates. Since this variant was discovered recently, there has not yet been any in-vivo studies reported.

The most recently discovered allelic variant, CYP2C9.6, occurs as a result of an adenine base pair deletion at nucleotide 818 , creating a premature stop codon (Kidd et al., 2001). The outcome of which is a truncated protein that is inactive. This variant was found in one African-American subject who experienced an adverse reaction to phenytoin due to reduced clearance of the drug from the body (Kidd et al., 2001). Further genotyping studies have revealed that one of 158 alleles identified in 79 AfricanAmerican subjects contained the deletion (Kidd et al., 2001). No subjects in a French Caucasian population were found to have contained the mutation (Kidd et al., 2001). Further investigation of the prevalence of this variant in the population is needed.

A number of substrates have been used as in-vitro and in-vivo probes for CYP2C9 activity. Good probe substrates are readily metabolized, stable enough for quantification, and are only metabolized by one $\mathrm{P} 450$ isoform, which is especially important when dealing with in-vivo studies or in-vitro studies using human liver microsomes that contain all of the isoforms. Flurbiprofen is a NSAID that is used as a CYP2C9 probe substrate in both in-vivo and in-vitro studies, and has been shown to be 4'-hydroxylated solely by CYP2C9 and exhibits Michaelis-Menten type kinetics (Tracy et al., 1995; Tracy et al., 1996). The NSAID, naproxen, is another probe substrate often used for studying CYP2C9 activity. Although CYP1A2 is involved in the metabolism of naproxen, CYP2C9 is mostly responsible (Miners et al., 1996;Tracy et al., 1997). In 
human liver microsomes, a biphasic kinetic profile is evident suggesting a role for more than one P450 isoform (Tracy et al., 1997). However, when incubated with purified CYP2C9 enzyme, naproxen metabolism still exhibits a biphasic kinetic profile suggesting that more than one productive binding region exists for naproxen within the enzyme, making naproxen an interesting compound for study (Hutzler et al., 2001;Korzekwa et al., 1998; Tracy et al., 2002).

Not only is CYP2C9 mediated metabolism affected by amino acid substitutions of the allelic variants, but also by chemical effectors. Normally when more than one substrate is present, inhibition occurs. However, the opposite is true when dapsone is coincubated with certain substrates and CYP2C9. Dapsone is an antibacterial agent used mainly for the treatment of Pneumocystis carinii infections in AIDS patients. The presence of dapsone increases the CYP2C9 mediated metabolism of flurbiprofen, naproxen, and piroxicam metabolism (Hutzler et al., 2001;Hutzler et al., 2002;Korzekwa et al., 1998). The activation, or increase in metabolism due to the presence of another substrate is also known as positive heterotropic cooperativity. This phenomenon has only recently been described and has been studied in CYP3A4 as well as CYP2C9 (Galetin et al., 2002;Nakamura et al., 2002; Shou et al., 1994).

While the effects of allelic variants and the presence of dapsone on CYP2C9 mediated metabolism have been studied, the effects of dapsone activation on the allelic variants of CYP2C9 have not. We hypothesized that since the CYP2C9 allelic variants show differential rates of metabolism, they will also show differential degrees of dapsone activation. Six concentrations of dapsone and six concentrations of substrate, either flurbiprofen or naproxen, were co-incubated with purified CYP2C9.1, CYP2C9.2, 
CYP2C9.3, or CYP2C9.5 that were reconstituted with dilauroylphosphatidylcholine vesicles, NADPH Reductase, and cytochrome b5. This resulted in six kinetic profiles containing six data points each, and the estimation of six sets of kinetic parameters (i.e. Vmax and $\mathrm{Km}$ ) for each variant form and substrate studied. Enzyme efficiencies $(\mathrm{Vmax} / \mathrm{Km})$ were calculated from the estimates and were used as the basis of comparison for dapsone activation. 


\section{CHAPTER II}

2. Materials and Methods 
Chemicals and Reagents. S-Flurbiprofen, 4'-hydroxyflurbiprofen, and 2-fluoro 4biphenyl acetic acid were gifts from Pharmacia Corporation (Kalamazoo, MI). SNaproxen and desmethylnaproxen were gifts from Syntex Laboratories (Palo Alto, CA). Octyl-Sepharose CL-4B, sodium cholate, dapsone, antibiotic and antimycotic solution containing 10,000 units of penicillin $\mathrm{G}, 10 \mathrm{mg}$ streptomycin, and $25 \mu \mathrm{g}$ amphotericin B per mL, NADPH, dilauroylphosphatidylcholine, and PMSF were purchased from SigmaAldrich (St. Louis, MO). Acetonitrile, potassium phosphate, EDTA, and dithiothreitol were purchased from Fisher Scientific (Pittsburgh, PA). Emulgen 911 was obtained from Kao Atlas (Japan). Ceramic hydroxyapatite Type I was purchased from Bio-Rad Laboratories (Hercules, CA). Hy-Q SFX insect media was purchased from HyClone Laboratories (Logan, UT). All other chemicals were purchased from commercial sources and were of the highest purity available.

Mutagenesis and Vector Construction. The construction and amplification of the baculovirus vectors necessary for production of the recombinant CYP2C9 alellic variants were carried out according to established methods (Dickmann et al., 2001;Haining et al., 1996) and the vectors were provided to us by Dr. Robert Haining and Dr. Allan Rettie.

Enzyme Expression. The expression of recombinant human CYP2C9 and variants was performed according to the previous methods of Haining et al., (Haining et al., 1996). Trichoplusia ni cells were grown with Hy-Q SFX insect media supplemented with $10 \%$ heat inactivated fetal bovine serum and $1 \%$ antibiotic antimycotic solution on $10 x 100 \mathrm{~mm}$ culture dishes at $27^{\circ} \mathrm{C}$. For expressing CYP2C9 and its variants, the T. ni cells were scaled up to $250 \mathrm{~mL}$ suspension cultures in $2 \mathrm{~L}$ flasks and spun on a magnetic stir plate at $250 \mathrm{RPM}$ to prevent cell clumping and ensure an adequate oxygen supply. 
When the viable cell density reached $1-2 \times 10^{6}$ cells $/ \mathrm{mL}$, the cells were infected with the appropriate baculovirus. Two days post infection, supplemental hemin, dissolved in $\mathrm{NH}_{4} \mathrm{OH}$, was added to the suspension cultures to give a final concentration of $1-5 \mu \mathrm{g} / \mathrm{mL}$. Three days after infection, the suspension culture was centrifuged at 3,000 RPM for 20 minutes. The pelleted cells were then washed once with storage buffer and re-centrifuged at 3,000 RPM for 15 minutes. The cell pellet was then stored at $-80^{\circ} \mathrm{C}$ until purification.

Enzyme Purification. The purification of recombinant human CYP2C9 and variants was performed according to the previous methods of Haining et al., (Haining et al., 1996). All steps were carried out at $4^{\circ} \mathrm{C}$. The cell pellet was homogenized with 5-10 passes of a glass/Teflon homogenizer with Solubilization Buffer [50mM potassium phosphate pH 7.4, 20\% glycerol, 1 mM EDTA, 0.4 mM PMSF, 1 mM DTT, 1.0\% sodium cholate] at a concentration of $1 \mathrm{~mL}$ buffer/nmol P450. The homogenized pellet was then stirred for 60 minutes to further solubilize the membrane-bound P450's. The mixture was then centrifuged at $100,000 \mathrm{~g}$ for 60 minutes to remove insoluble cell debris. The supernatant was then loaded at $60 \mathrm{~mL} / \mathrm{hr}$ onto a $2.5 \times 20 \mathrm{~cm}$ Octyl-Sepharose CL-4B (1 mL/nmol P450) column pre-equilibrated with at least 10 column volumes of Buffer A [50 mM potassium phosphate $\mathrm{pH}$ 7.4, 20\% glycerol, $1 \mathrm{mM}$ EDTA, $0.2 \mathrm{mM}$ PMSF, $1 \mathrm{mM}$ DTT, $0.1 \mathrm{M} \mathrm{NaCl}, 0.5 \%$ sodium cholate]. The P450 appeared on the column as a red band. The column was then washed with Buffer A + 0.1\% Emulgen 911 until the red band moved approximately $2 / 3$ down the column. The P450 was then eluted with Buffer $\mathrm{A}+1.0 \%$ Emulgen 911 . The P450 containing fractions were collected and then diluted 2 fold with Buffer B [25 mM potassium phosphate $\mathrm{pH}$ 7.4, 20\% glycerol, $1 \mathrm{mM}$ EDTA, 0.5 mM DTT, 0.5\% Emulgen 911] and dialyzed overnight against Buffer B. The dialyzed 
fractions were then loaded at $80 \mathrm{ml} / \mathrm{hr}$ onto a $1.0 \times 15 \mathrm{~cm}$ ceramic hydroxyapatite (1 $\mathrm{mL} / 100$ nmol P450) column pre-equilibrated with Buffer B. The P450 appeared as a red band on the hydroxyapatite. The column was then washed with Buffer C [ $25 \mathrm{mM}$ potassium phosphate $\mathrm{pH}$ 7.4, 20\% glycerol, $1 \mathrm{mM}$ EDTA, $0.5 \mathrm{mM}$ DTT, $0.1 \%$ sodium cholate] until the UV absorbance at $280 \mathrm{~nm}$ was less than 0.2 in order to ensure the removal of the Emulgen 911. The P450 was then eluted with Buffer D $[500 \mathrm{mM}$ potassium phosphate $\mathrm{pH} 7.4,20 \%$ glycerol, $1 \mathrm{mM}$ EDTA, $0.5 \mathrm{mM}$ DTT]. The fractions were collected, pooled and dialyzed overnight against two changes of Buffer E [50 mM potassium phosphate $\mathrm{pH} 7.4,20 \%$ glycerol, $1 \mathrm{mM}$ EDTA]. $500 \mu \mathrm{L}$ aliquots were then stored in microcentrifuge tubes at $-80^{\circ} \mathrm{C}$ until further use.

Enzyme Reconstitution. Dilauroylphosphatidylcholine was weighed out and hydrated in $50 \mathrm{mM}$ potassium phosphate buffer $\mathrm{pH} 7.4$ to make a $1 \mathrm{mg} / \mathrm{mL}$ solution. The solution sat for one hour in order to allow for formation of lipid vesicles. The vesicles were then extruded through a $200 \mathrm{~nm}$ pore-sized membrane to make the vesicles uniform in size. The CYP2C9 variant of choice and P450 NADPH Reductase were then gently mixed in a 1:2 ratio in a microcentrifuge tube with the end of the pipette tip and allowed to sit for five minutes. The lipid vesicles were then added and the mixture again was allowed to equilibrate for five minutes. Finally, cytochrome b5 was added in a 1:1 ratio with the P450 and the entire mixture was left to sit for five minutes. The mixture was then given a short centrifugation in order to get any of the reconstituted enzyme mixture off the sides of the tube and into the rest of the solution. The enzyme prep was then added to the incubation mixture containing substrate and effector. 
Incubation Conditions. Incubations were carried out according to the previous methods of Tracy et al., (Tracy et al., 2002). Incubation mixtures contained 5-20 pmol of purified enzyme, NADPH reductase, and cytochrome $b_{5}$ in a 1:2:1 ratio, reconstituted with dilauroylphosphatidylcholine vesicles extruded through a $200 \mathrm{~nm}$ pore sized membrane. Six concentrations of substrate, either 2-300 $\mu \mathrm{M}$ (S)-flurbiprofen or 10-1800 $\mu \mathrm{M}$ (S)-naproxen, were incubated with six concentrations of dapsone, $0-100 \mu \mathrm{M}$, for 20 minutes at $37^{\circ} \mathrm{C}$ in $50 \mathrm{mM}$ potassium phosphate buffer $\mathrm{pH} 7.4$ at a final volume of 200 $\mu \mathrm{L}$. The reactions were initiated by the addition of $1 \mathrm{mM}$ NADPH after a 3 minute preincubation. The reactions were quenched by the addition of $200 \mu \mathrm{L}$ acetonitrile containing internal standard, $180 \mathrm{ng} / \mathrm{mL}$ of 2-fluoro-4-biphenylacetic acid for (S)flurbiprofen or $500 \mathrm{ng} / \mathrm{mL}$ of 2-fluoro-4-biphenylacetic acid for (S)-naproxen. Following quenching, $40 \mu \mathrm{L}$ of half strength $\mathrm{H}_{3} \mathrm{PO}_{4}$ was added to the reaction mixtures for both substrates. Samples were then centrifuged at 10,000 rpm for 4 minutes, placed into autosampler vials, and 5-50 $\mu \mathrm{L}$ were injected onto the HPLC system.

HPLC Analysis. The HPLC system consisted of a Waters 501 pump module, a Waters 717 autosampler, and a Waters 470 fluorescence detector. For both metabolites, the mobile phase was pumped through a Brownlee Spheri-5 $\mathrm{C}_{18} 4.6-\mathrm{x}$ 100-mm column at $1 \mathrm{~mL} /$ minute. For quantification of 4 '-hydroxyflurbiprofen, the detector was set at an excitation wavelength of $260 \mathrm{~nm}$ and an emission wavelength of $320 \mathrm{~nm}$, and the mobile phase consisted of 45:55 acetonitrile: $20 \mathrm{mM}$ potassium phosphate $\mathrm{pH}$ 3.0. The retention times for 4'-hydroxyflurbiprofen and the internal standard were approximately 2.6 and 5.6 minutes, respectively. For quantification of desmethylnaproxen, the detector was set at an excitation wavelength of $230 \mathrm{~nm}$ and an emission wavelength of $340 \mathrm{~nm}$, and the 
mobile phase consisted of 40:60 acetonitrile:20 mM potassium phosphate $\mathrm{pH}$ 3.0. The retention times for desmethylnaproxen and the internal standard were approximately 2.3 and 9.6 minutes, respectively.

Data Analysis. Kinetic parameters for the substrates were estimated by nonlinear regression analysis using Sigma Plot 7.0. Data were fit to the Michaelis-Menten equation, $v=\frac{\left(V_{\max } \cdot S\right)}{K_{m}+S}$, a linear equation, $v=\left(C L_{\text {int }}\right) S$, or a biphasic equation, $v=\frac{\left(V_{m 1} \cdot S\right)+\left(C L_{\mathrm{int}} \cdot S^{2}\right)}{K_{m 1}+S}$. 


\section{CHAPTER III}

\section{Dapsone Activation of CYP2C9 Allelic Variants}




\subsection{Introduction}

Differences in metabolism among allelic variants of cytochromes P450 are becoming increasingly important as knowledge is being gained about the in-vitro and invivo consequences of the single nucleotide polymorphisms which result in amino acid substitutions. One polymorphic $\mathrm{P} 450$ of importance is CYP2C9, because it is involved in the metabolism of a wide range of substrates, including S-warfarin, phenytoin, tolbutamide, as well as a number of NSAIDS (Leemann et al., 1993;Rettie et al., 1992;Rodrigues et al., 1996; Tracy et al., 1996; Veronese et al., 1991a;Zhao et al., 1992). When assessing the effects of CYP2C9 allelic variants, the substrates of most concern are the low therapeutic index drugs, S-warfarin and phenytoin, because individuals expressing an allelic variant may exhibit reduced clearance of these agents (Furuya et al., 1995; Hashimoto et al., 1996;Rettie et al., 1994).

Six allelic variants of CYP2C9 have been described to date, including CYP2C9.1 (wild type), CYP2C9.2 (R144C), CYP2C9.3 (I359L), CYP2C9.4 (I359T), CYP2C9.5 (D360E), and CYP2C9.6 (818delA). Of the six, the most well characterized are CYP2C9.1, CYP2C9.2, and CYP2C9.3. Compared to the wild type enzyme, CYP2C9.2 and CYP2C9.3, found mostly in the Caucasian population, exhibit reduced metabolism of a number of substrates (Haining et al., 1996;Steward et al., 1997;Takanashi et al., 2000; Yamazaki et al., 1998). The CYP2C9.2 variant enzyme appears to reduce the Vmax of substrate turnover but have little effect on Km (Yamazaki et al., 1998). However, with the CYP2C9.3 allele, $\mathrm{Km}$ is increased for all studied substrates whereas Vmax is decreased (Takanashi et al., 2000). Limited information is available concerning the rare variant, CYP2C9.4, which was found in a Japanese epileptic patient, but this 
patient required a lower than normal dose of phenytoin (Ieiri et al., 2000). CYP2C9.5, which has been shown to occur in the African-American and African populations, has been associated with reductions in in-vitro intrinsic clearance of a number of substrates, including lauric acid, S-warfarin, diclofenac, flurbiprofen, naproxen, and piroxicam. (Dickmann et al., 2001; Tracy et al., 2002) but in vivo consequences of this amino acid change are to date unknown. Interestingly, this allele was found in $1 \%$ of a Tanzanian population but not in an Ethiopian population (Yasar et al., 2002). The most recently described variant, CYP2C9.6, was identified in an African-American subject exhibiting phenytoin toxicity (Kidd et al., 2001). Since this protein is truncated as a result of a premature stop codon, it is inactive.

Dapsone has been shown to activate the metabolism of CYP2C9 substrates, such as flurbiprofen, naproxen, and piroxicam (Hutzler et al., 2001;Hutzler et al., 2002;Korzekwa et al., 1998). This activation results in a decrease in the $\mathrm{Km}$ and an increase in the Vmax of the CYP2C9 enzyme causing an increase in efficiency of the enzyme and substrate turnover. Since amino acid changes in key residues, such as those found in the allelic variants, alter rates of metabolism, these substitutions may also affect the degree of activation observed since it has been proposed that the activator must also bind within the enzyme active site to cause its effect. However, the degree of dapsone mediated activation of CYP2C9 allelic variants has not been assessed. Using flurbiprofen and naproxen as probe substrates, the effects of dapsone on CYP2C9.1, CYP2C9.2, CYP2C9.3, and CYP2C9.5 mediated metabolism were studied to determine whether the degree of enzyme activation is dependent on the enzyme allelic variant being studied. 


\subsection{Materials and Methods}

Chemicals and Reagents. S-Flurbiprofen, 4'-hydroxyflurbiprofen, and 2-fluoro 4biphenyl acetic acid were gifts from Pharmacia Corporation (Kalamazoo, MI). SNaproxen and desmethylnaproxen were gifts from Syntex Laboratories (Palo Alto, CA). Dapsone, NADPH, and dilauroylphosphatidylcholine were purchased from SigmaAldrich (St. Louis, MO). Acetonitrile and potassium phosphate were purchased from Fisher Scientific (Pittsburgh, PA). Human P450 oxidoreductase and human cytochrome b5 were purchased from Pan Vera LLC (Madison, WI). All other chemicals were purchased from commercial sources and were of the highest purity available.

Enzyme Construction and Expression. Mutagenesis and vector construction of the wild type enzyme, the R144C (*2), I359L (*3), and the D360E (*5) variants were carried out according to previously established methods (Dickmann et al., 2001;Haining et al., 1996). Proteins were expressed using a baculovirus mediated expression system in Trichoplusia ni cells and carried out according to the methods of Haining et al., (1996). Protein purification was performed as described previously (Dickmann et al., 2001).

Incubation Conditions. Incubations were carried out according to the methods of Tracy et al., (2002). Incubation mixtures contained 5-20 pmol of purified enzyme, NADPH reductase, and cytochrome $b_{5}$ in a 1:2:1 ratio, reconstituted with dilauroylphosphatidylcholine vesicles extruded through a $200 \mathrm{~nm}$ pore sized membrane. Six concentrations of substrate, either 2-300 $\mu \mathrm{M}$ (S)-flurbiprofen or 10-1800 $\mu \mathrm{M}$ (S)naproxen, were incubated with six concentrations of dapsone, $0-100 \mu \mathrm{M}$, for 20 minutes at $37^{\circ} \mathrm{C}$ in $50 \mathrm{mM}$ potassium phosphate buffer $\mathrm{pH} 7.4$ in a final volume of $200 \mu \mathrm{L}$. The 
reactions were initiated by the addition of $1 \mathrm{mM}$ NADPH after a 3 minute preincubation. Reactions were quenched by the addition of $200 \mu \mathrm{L}$ acetonitrile containing internal standard, $180 \mathrm{ng} / \mathrm{mL}$ of 2-fluoro-4-biphenylacetic acid for (S)-flurbiprofen or $500 \mathrm{ng} / \mathrm{mL}$ of 2-fluoro-4-biphenylacetic acid for (S)-naproxen. Following quenching, 40 $\mu \mathrm{L}$ of half strength $\mathrm{H}_{3} \mathrm{PO}_{4}$ was added to the reaction mixtures for both substrates. Samples were then centrifuged at 10,000 rpm for 4 minutes, placed into autosampler vials, and 5-50 $\mu \mathrm{L}$ injected onto the HPLC system.

HPLC Analysis. HPLC analysis was conducted as described previously (Tracy et al., 2002). The HPLC system consisted of a Waters 501 pump module, a Waters 717 autosampler, and a Waters 470 fluorescence detector. For both metabolites, the mobile phase was pumped through a Brownlee Spheri-5 $\mathrm{C}_{18} 4.6$ x $100 \mathrm{~mm}$ column at 1 $\mathrm{mL} /$ minute. For quantification of $4^{\prime}$-hydroxyflurbiprofen, the detector was set at an excitation wavelength of $260 \mathrm{~nm}$ and an emission wavelength of $320 \mathrm{~nm}$, and the mobile phase consisted of 45:55 acetonitrile:20 mM potassium phosphate $\mathrm{pH}$ 3.0. The retention times for 4'-hydroxyflurbiprofen and the internal standard were approximately 2.6 and 5.6 minutes, respectively. For quantification of desmethylnaproxen, the detector was set at an excitation wavelength of $230 \mathrm{~nm}$ and an emission wavelength of $340 \mathrm{~nm}$, and the mobile phase consisted of 40:60 acetonitrile:20 mM potassium phosphate $\mathrm{pH}$ 3.0. The retention times for desmethylnaproxen and the internal standard were approximately 2.3 and 9.6 minutes, respectively.

Data Analysis. Kinetic parameters for the substrates were estimated by nonlinear regression analysis, using Sigma Plot 7.0. Data were fit to the Michaelis-Menten 
equation, $v=\frac{\left(V_{\max } \cdot S\right)}{K_{m}+S}$, a linear equation, $v=\left(C L_{\mathrm{int}}\right) S$, or a biphasic equation $v=\frac{\left(V_{m 1} \cdot S\right)+\left(C L_{\mathrm{int}} \cdot S^{2}\right)}{K_{m 1}+S}$

\subsection{Results}

Figure 3.1 depicts the best fit of the data to the standard Michaelis-Menten equation for dapsone activation of flurbiprofen 4'-hydroxylation by CYP2C9.1, CYP2C9.2, CYP2C9.3, and CYP2C9.5. Kinetic parameter estimates for the fits can be found in Table 3.1. In the absence of dapsone, CYP2C9.1 exhibited the highest activity of flurbiprofen 4'-hydroxylation, followed by CYP2C9.2, CYP2C9.5, and CYP2C9.3. In all of the variants, dapsone increased the metabolism of flurbiprofen. The Vmax was increased roughly 2-fold while the Km was decreased 4-fold in the presence of $100 \mu \mathrm{M}$ dapsone as compared to the absence of dapsone in CYP2C9.1. Similar trends were noted for the other variants, although to varying degrees. These differences in increase of enzymatic efficiency $(\mathrm{Vmax} / \mathrm{Km})$ among the different allelic variants in the presence of dapsone are presented in Table 3.3.

Naproxen demethylation was also activated by the presence of dapsone in all of the allelic variants tested (Figure 3.2). In the cases of CYP2C9.1 in the presence of $0-5$ $\mu \mathrm{M}$ dapsone and CYP2C9.2 in the presence of $0-2 \mu \mathrm{M}$ dapsone, the resulting data were best fit to a biphasic equation, resulting in estimates of Vmax, $\mathrm{Km}$ and CLint describing the biphasic nature of the data. For CYP2C9.3 and CYP2C9.5 in the presence of $0-2 \mu \mathrm{M}$ dapsone the data were best fit to a linear equation with CLint representing the slope of the 
line, since no hyperbolic character was noted within the substrate concentration range studied. The remaining data for each of the variants in the presence of $10-100 \mu \mathrm{M}$ dapsone (CYP2C9.1) or 5-100 $\mu \mathrm{M}$ dapsone (CYP2C9.2, CYP2C9.3, CYP2C9.5) were best fit to the Michaelis-Menten equation. Kinetic parameter estimates are represented in Table 3.2. As with flurbiprofen, dapsone increased the Vmax of naproxen demethylation, while the $\mathrm{Km}$ was decreased for all variants. As implied above, the rates of naproxen demethylation became increasingly hyperbolic with increasing dapsone concentration in the case of all CYP2C9 variants. As illustrated with flurbiprofen, the efficiency (Vmax/Km) of CYP2C9 mediated naproxen demethylation increased to varying degrees depending on the allelic variant studied (Table 3.3).

\subsection{Discussion}

Many factors can confound formulation of accurate in-vitro/in-vivo correlations, including atypical kinetics, such as positive heterotropic cooperativity or activation, as well as the effects of allelic variants on metabolism. CYP2C9 exhibits all of these characteristics. We have previously demonstrated that dapsone can activate CYP2C9 mediated metabolism of flurbiprofen and naproxen (Hutzler et al., 2001) and that allelic variants of CYP2C9 exhibit lower substrate turnover (Tracy et al., 2002). However, whether allelic variants exhibit differential activation by enzyme effectors such as dapsone was unknown. We hypothesized that because allelic variants of this enzyme exhibit reduced turnover of substrate, they would also be affected differentially by the effector, dapsone, and thus exhibit differential activation of CYP2C9 mediated metabolism as compared to wild type enzyme. Thus we studied the effects of dapsone on 
flurbiprofen and naproxen metabolism by R144C (CYP2C9.2), I359L (CYP2C9.3), and the D360E (CYP2C9.5) variants of CYP2C9 and compared the results to the wild type enzyme. Our results suggest that the substitutions alter the degree of dapsone activation, as measured by the increase in enzyme efficiency $(\mathrm{Vmax} / \mathrm{Km})$ of flurbiprofen 4'hydroxylation and naproxen demethylation to different extents, not only compared to the wild type enzyme, but compared to one another as well.

Flurbiprofen was used to examine dapsone activation of CYP2C9 allelic variants because its metabolism has been shown to be activated by dapsone with the wild type enzyme and it exhibits typical Michaelis-Menten kinetics. In the absence of dapsone, CYP2C9.2, CYP2C9.3, and CYP2C9.5 exhibited a Vmax/Km ratio (measure of enzyme efficiency) for flurbiprofen 4 '-hydroxylation that was 70\%, 4\%, and 10\% of CYP2C9.1 activity, respectively. In all variants, $\mathrm{Km}$ was reduced and Vmax increased in the presence of dapsone, although to different extents depending on the allelic variant being studied. In the case of flurbiprofen hydroxylation, the efficiency of the CYP2C9.3 variant was increased greater than 6 -fold more in the presence of dapsone than was the efficiency of the wild type enzyme whereas the efficiency of the CYP2C9.2 and CYP2C9.5 variants were increased 4.5- and 3-fold more than was the wild type enzyme. These findings suggest that these amino acids play a key role not only in flurbiprofen hydroxylation but also are in some way involved in the extent of dapsone activation. However, the role they play in activation and increase in enzyme efficiency is not clearly defined. The substituted residues may change substrate and/or effector binding directly, alter folding of the protein or increase the efficiency of the enzyme through other unknown mechanisms. 
Naproxen was also tested for differential effects of dapsone activation of the allelic variants of CYP2C9, because naproxen exhibits atypical kinetics presumably due to occupation of multiple binding regions within the CYP2C9 active site (Hutzler et al., 2001;Korzekwa et al., 1998). Additionally, co-incubation with dapsone results in the conversion of the biphasic kinetic profile to a hyperbolic kinetic profile (Hutzler et al., 2001). In the absence of dapsone, CYP2C9.1 and CYP2C9.2 mediated naproxen demethylation was biphasic in nature, and CYP2C9.3 and CYP2C9.5 mediated naproxen demethylation had a linear kinetic profile over the concentration ranges that were studied. Because of the differences in kinetic profiles observed, direct comparison of kinetic parameters among the allelic variants is complicated. However, in each of the variants and wild type enzyme, at $10 \mu \mathrm{M}$ dapsone concentrations and higher, the data were best fit with a hyperbolic equation (i.e., normalization of kinetics) allowing comparison of kinetic parameters. As with flurbiprofen, dapsone increased the Vmax and reduced the $\mathrm{Km}$ for naproxen demethylation in all cases. Again, however, there are differences in efficiency increase among the variants and the rank order was different than observed with flurbiprofen hydroxylation. For naproxen demethylation, the CYP2C9.5 variant exhibited the greatest increase in enzyme efficiency $(\mathrm{Vmax} / \mathrm{Km})$ with lesser increases noted with the CYP2C9.2 and CYP2C9.3 variants. However, it should be re-emphasized that these comparisons are somewhat confounded by the change in kinetic profile. This normalization of kinetics has been hypothesized to be due to dapsone filling one of the binding regions in the active site of the enzyme and thus causing naproxen to occupy only one site (instead of the usual two) and now behaving in a hyperbolic kinetic manner (Hutzler et al., 2001). This "normalization" of kinetic profile has also been observed 
with the CYP3A4 enzyme by Nakamura and colleagues who demonstrated that androstenedione could activate CYP3A4 mediated carbamazepine metabolism and convert the kinetic profile from a sigmoidal to a hyperbolic kinetic form (Nakamura et al., 2002).

CYP2C9.2 has been associated with reduced metabolism both in-vitro and invivo, mainly by reducing the Vmax, with little effect on $\mathrm{Km}$. Because of this, amino acid position 144 is not thought to be in the active site of the enzyme, though the publishing of the CYP2C9 crystal structure may provide additional insight. Crespi and Miller have implicated this substitution in reducing the interaction of the P450 with NADPH reductase, which is essential for electron flow necessary for the oxidation of substrates (Crespi and Miller, 1997). This effect could be due to the fact that the side chain of arginine is charged at $\mathrm{pH} 7.4$, making an electrostatic contact with the reductase, whereas the cysteine side chain is polar, but not charged. However, in this study, the presence of dapsone decreased the $\mathrm{Km}$ for both flurbiprofen 4'-hydroxylation and naproxen demethylation for CYP2C9.2. Thus, it is unclear whether this residue does in fact play a role in substrate or effector binding, causes a change in protein folding or in conjunction with dapsone inhibits enhanced electron flow through the enzyme.

The CYP2C9.3 variant has a leucine residue in place of an isoleucine residue at amino acid position 359 and the CYP2C9.5 variant contains a glutamate in place of an aspartate at position 360 . Both position 359 and 360 have been purported to be in substrate recognition site (SRS) 5 and may play a role in substrate binding (Gotoh, 1992). Though these amino acid changes appear minor, the substitution may alter the size or shape of the binding pocket and cause differences in steric hinderance compared to the 
wild type protein, resulting in reductions in the metabolism of substrates or effector binding, or the protein may take on a different shape as a result of a variation in protein folding with the substituted amino acid. Regardless, these variants appear to be affected differentially by dapsone as compared to the wild type enzyme and thus play a role in activation of the enzyme.

Not only were the effects of dapsone activation variant specific, they were also substrate specific. Effects of allelic variants on metabolism have been shown to be substrate specific (Takanashi et al., 2000; Tracy et al., 2002). In this study, flurbiprofen 4'-hydroxylation was minimally activated in the presence of 2-10 $\mu \mathrm{M}$ dapsone, while naproxen demethylation was activated to a greater extent at lower dapsone concentrations. Also, the maximal velocity of naproxen demethylation was increased to a level double that of flurbiprofen 4'-hydroxylation with the exception of CYP2C9.2, which was similar. By testing a number of steroids with a variety of substrates, Nakamura et. al., demonstrated that there are substrate specific differences in effects of endogenous steroids on CYP3A4 mediated metabolism (Nakamura et al., 2002). Similarly, Galetin et. al., showed that quinidine differentially activated or inhibited CYP3A4 mediated metabolism depending on the substrate used (Galetin et al., 2002). We have shown that dapsone activates CYP2C9 mediated metabolism to different extents based on the substrate being studied.

It is also interesting to note that in all cases, dapsone was able to activate the turnover of substrate to a level similar to or greater than the level achieved by CYP2C9.1 in the absence of dapsone. As stated previously, CYP2C9.2, CYP2C9.3, and CYP2C9.5 exhibited a $V \max / \mathrm{Km}$ ratio that was $70 \%, 4 \%$, and $10 \%$, respectively, that of the wild 
type enzyme in the absence of dapsone. However, when comparing the Vmax/Km ratio for flurbiprofen 4'-hydroxylation for CYP2C9.2, CYP2C9.3, and CYP2C9.5 in the presence of $100 \mu \mathrm{M}$ dapsone to CYP2C9.1 in the absence of dapsone, they have a $\mathrm{Vmax} / \mathrm{Km}$ ratio that is $2190 \%, 188 \%$, and $220 \%$ of the wild type enzyme in the absence of dapsone. For naproxen demethylation, it is more difficult to compare the kinetic parameters due to the different kinetic profiles for the absence and presence of dapsone. However, from Figure 3.2, it is clear that naproxen demethylation is increased to a level greater than that of the wild type for all of the allelic variants studied, especially at lower concentrations of naproxen. Whether this ability to convert a "poor" metabolizer to an extensive metabolizer state in vitro can be translated to the in vivo situation remains to be studied. However, it does raise intriguing possibilities in terms of the potential ability to normalize patients with respect to drug metabolism capacity.

In summary, the ability of dapsone to activate CYP2C9 mediated metabolism is both allelic variant and substrate dependent. Dapsone co-incubation reduces the Km and increase the Vmax of both flurbiprofen 4'-hydroxylation and naproxen demethylation resulting in an increase in the overall efficiency of the enzyme with greater effects being noted in the allelic variants as compared to wild type enzyme. These findings demonstrate that the degree of drug-drug interaction may be influenced by an individual's genotype. 


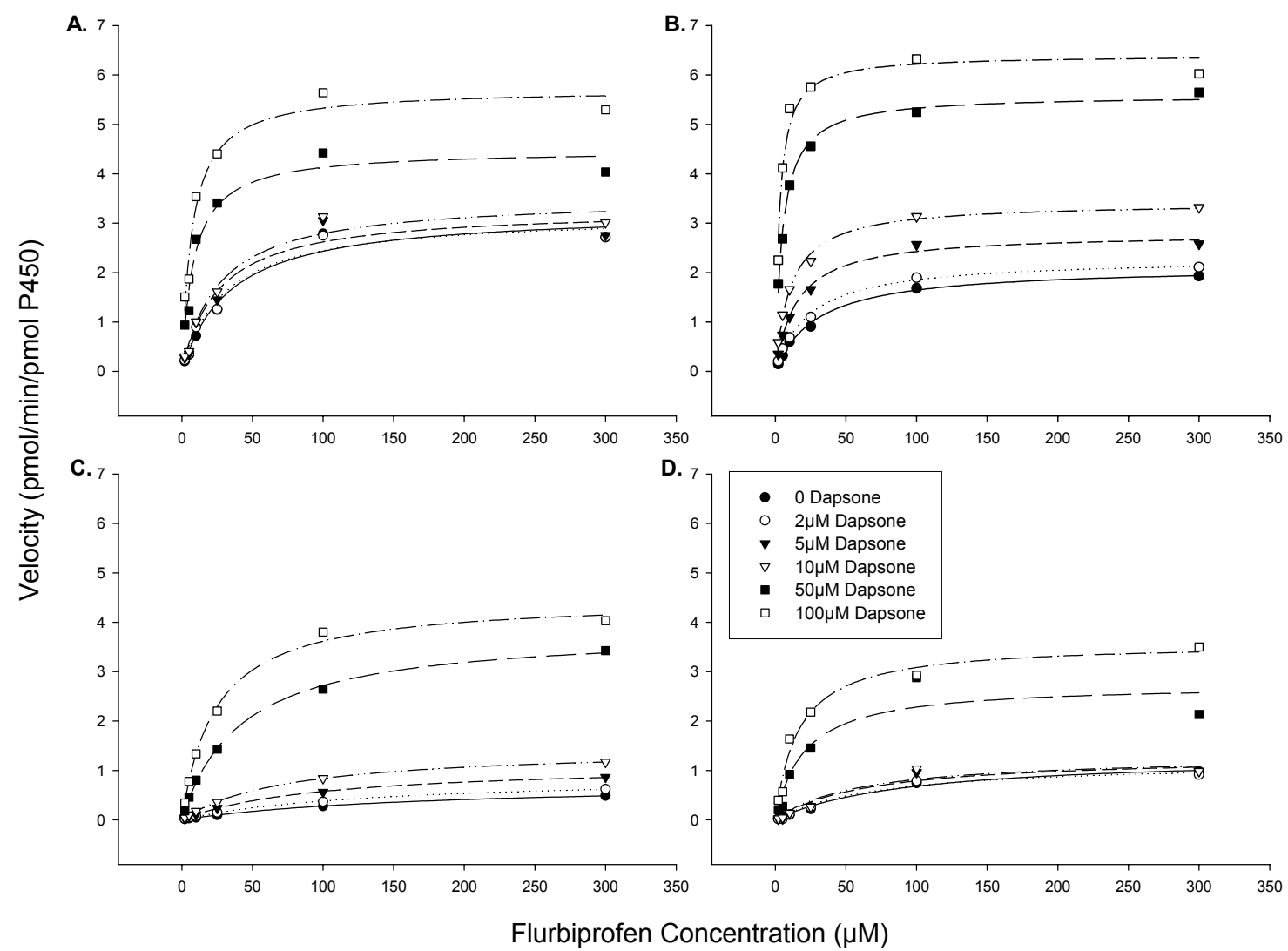

Figure 3.1 Dapsone activation of flurbiprofen 4'-hydroxylation by (A) CYP2C9.1, (B) CYP2C9.2, (C) CYP2C9.3, and (D) CYP2C9.5. 


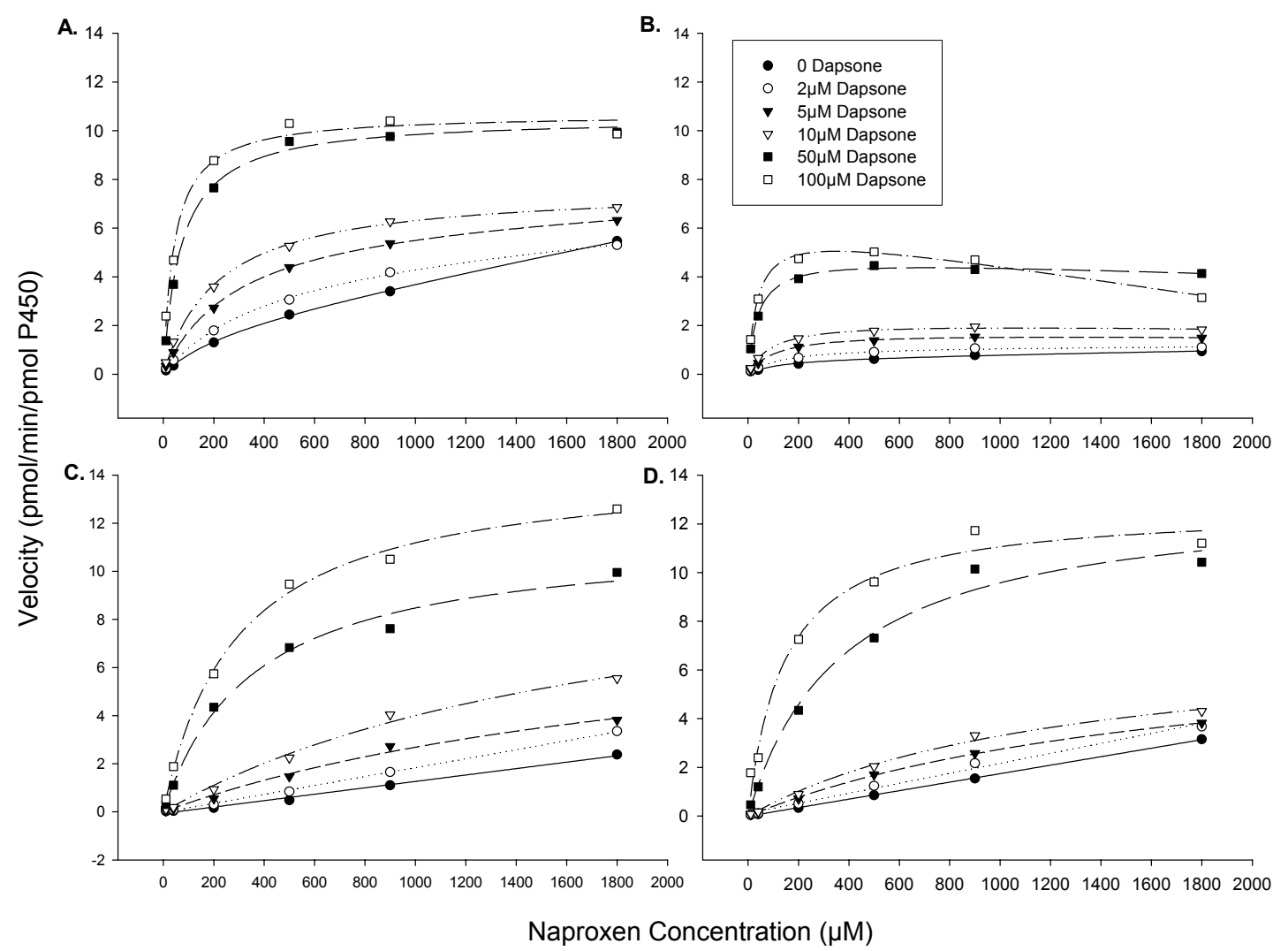

Figure 3.2 Dapsone activation of naproxen demethylation by (A) CYP2C9.1, (B) CYP2C9.2, (C) CYP2C9.3, and (D) CYP2C9.5. 
Table 3.1 Kinetic Parameter Estimates for Flurbiprofen 4'-hydroxylation.

\begin{tabular}{|c|c|c|c|c|c|c|c|c|c|c|c|c|}
\hline \multirow[b]{2}{*}{ Dapsone } & \multicolumn{3}{|c|}{ CYP2C9.1 } & \multicolumn{3}{|c|}{ СYР2C9.2 } & \multicolumn{3}{|c|}{ СYP2C9.3 } & \multicolumn{3}{|c|}{ CYP2C9.5 } \\
\hline & $V_{\max }$ & $K_{m}$ & $V_{\max } / K_{m}$ & $V_{\max }$ & $K_{m}$ & $V_{\max } / K_{m}$ & $V_{\max }$ & $K_{m}$ & $V_{\max } / K_{m}$ & $V_{\max }$ & $K_{m}$ & $V_{\max } / K_{m}$ \\
\hline$\mu M$ & pmol/min/pmol P450 & $\mu M$ & $\mu l /$ min/pmol P450 & pmol/min/pmol P450 & $\mu M$ & $\mu l / m i n / p m o l ~ P 450$ & pmol/min/pmol P450 & $\mu M$ & $\mu l / \min / p m o l ~ P 450$ & pmol/min/pmol P450 & $\mu M$ & ${ }_{\mu l / m i n} / \mathrm{pmol} P 450$ \\
\hline 0 & $3.25(0.28)$ & $33.5(9.35)$ & 0.10 & $2.13(0.06)$ & $29.2(2.68)$ & 0.07 & $0.76(0.03)$ & $170(15.7)$ & 0.004 & $1.32(0.11)$ & $93.1(21.1)$ & 0.01 \\
\hline 2 & $3.18(0.25)$ & $29.9(7.87)$ & 0.11 & $2.29(0.06)$ & $23.8(2.18)$ & 0.10 & $0.89(0.05)$ & $133(15.6)$ & 0.007 & $1.19(0.14)$ & $72.5(24.0)$ & 0.02 \\
\hline 5 & $3.29(0.32)$ & $25.6(8.64)$ & 0.13 & $2.80(0.09)$ & $15.2(1.74)$ & 0.18 & $1.13(0.03)$ & $96.1(7.19)$ & 0.012 & $1.30(0.20)$ & $63.7(28.6)$ & 0.02 \\
\hline 10 & $3.52(0.26)$ & $26.0(6.46)$ & 0.14 & $3.42(0.08)$ & $10.8(0.94)$ & 0.32 & $1.46(0.02)$ & $73.5(2.72)$ & 0.020 & $1.30(0.24)$ & $58.9(32.5)$ & 0.02 \\
\hline 50 & $4.48(0.27)$ & $8.48(2.01)$ & 0.53 & $5.59(0.11)$ & $5.00(0.41)$ & 1.12 & $3.85(0.08)$ & $41.4(2.66)$ & 0.093 & $2.75(0.40)$ & $20.3(10.4)$ & 0.14 \\
\hline 100 & $5.72(0.28)$ & $7.26(1.45)$ & 0.79 & $6.40(0.20)$ & $2.92(0.47)$ & 2.19 & $4.47(0.13)$ & $23.8(2.33)$ & 0.188 & $3.58(0.20)$ & $16.1(3.31)$ & 0.22 \\
\hline
\end{tabular}

Parameter estimates are reported as the estimate (standard error of the estimate) resulting from nonlinear regression of the data. 
Table 3.2 Kinetic Parameter Estimates for Naproxen Demethylation.

\begin{tabular}{|c|c|c|c|c|c|c|c|c|c|c|c|c|}
\hline \multirow[b]{2}{*}{ Dapsone } & \multicolumn{3}{|c|}{ CYP2C9.1 } & \multicolumn{3}{|c|}{ CYP2C9.2 } & \multicolumn{3}{|c|}{ CYP2C9.3 } & \multicolumn{3}{|c|}{ CYP2C9.5 } \\
\hline & $V_{\max }$ & $K_{m}$ & CLint & $V_{\max }$ & $K_{m}$ & CLint & $V_{\max }$ & $K_{m}$ & CLint & $V_{\max }$ & $K_{m}$ & CLint \\
\hline$\mu M$ & pmol/min/pmol P450 & $\mu M$ & $\mu l / m i n / p m o l ~ P 450$ & pmol/min/pmol P450 & $\mu M$ & $\mu l /$ min/pmol P450 & pmol/min/pmol P450 & $\mu M$ & $\mu l /$ min/pmol P450 & pmol/min/pmol P450 & $\mu M$ & $\mu l /$ min/pmol P450 \\
\hline 0 & $2.58(0.36)$ & $248(59.7)$ & $0.0020(0.0001)$ & $0.70(0.12)$ & $122(54.3)$ & $0.0002(0.0001)$ & - & - & $0.0013(0.0001)$ & - & - & $0.0017(0.0000)$ \\
\hline 2 & $5.40(0.97)$ & $408(120)$ & $0.0006(0.0004)$ & $1.15(0.12)$ & $132(35.5)$ & $0.0000(0.0001)$ & - & - & $0.0019(0.0000)$ & - & - & $0.0020(0.0001)$ \\
\hline 5 & $6.75(0.52)$ & $288(42.6)$ & $0.0003(0.0002)$ & - & - & - & - & - & - & - & - & - \\
\hline Dapsone & $V_{\max }$ & $K_{m}$ & $V_{\max } / K_{m}$ & $V_{\max }$ & $K_{m}$ & $V_{\max } / K_{m}$ & $V_{\max }$ & $K_{m}$ & $V_{\max } / K_{m}$ & $V_{\max }$ & $K_{m}$ & $V_{\max } / K_{m}$ \\
\hline$\mu M$ & pmol/min/pmol P450 & $\mu M$ & $\mu l /$ min/pmol P450 & pmol/min/pmol P450 & $\mu M$ & $\mu l /$ min/pmol P450 & pmol/min/pmol P450 & $\mu M$ & $\mu l /$ min/pmol P450 & pmol/min/pmol P450 & $\mu M$ & $\mu l /$ min/pmol P450 \\
\hline 5 & - & - & - & $1.64(0.04)$ & $94.3(11.2)$ & 0.017 & $8.85(1.73)$ & $2292(699)$ & 0.004 & $7.60(0.31)$ & $1768(120)$ & 0.004 \\
\hline 10 & $7.68(0.16)$ & $218(16.9)$ & 0.035 & $2.02(0.06)$ & $76.6(10.9)$ & 0.026 & $11.6(1.79)$ & 1909 (482) & 0.006 & $7.48(0.70)$ & $1266(223)$ & 0.006 \\
\hline 50 & $10.5(0.17)$ & $72.0(5.83)$ & 0.146 & $4.48(0.12)$ & $32.8(5.17)$ & 0.137 & $11.5(0.65)$ & $357(61.6)$ & 0.032 & $13.2(0.92)$ & $375(78.5)$ & 0.035 \\
\hline 100 & $10.7(0.32)$ & $44.2(7.49)$ & 0.242 & $4.52(0.46)$ & $17.7(11.7)$ & 0.255 & $14.4(0.45)$ & $289(29.7)$ & 0.050 & $12.7(0.74)$ & $144(35.9)$ & 0.088 \\
\hline
\end{tabular}

Parameter estimates are reported as the estimate (standard error of the estimate) resulting from nonlinear regression of the data.

Table is split into biphasic (CYP2C9.1 and CYP2C9.2) and linear (CYP2C9.3 and CYP2C9.5) fits of the data (TOP) and MichaelisMenten fits of the data (BOTTOM). 
Table 3.3 Percent increase in efficiency $(\mathrm{Vmax} / \mathrm{Km})$ of metabolism due to dapsone activation.

\begin{tabular}{ccc}
\hline Variant & Flurbiprofen & Naproxen \\
\hline CYP2C9.1 & $690 \%$ & $591 \%$ \\
CYP2C9.2 & $3029 \%$ & $1400 \%$ \\
CYP2C 9.3 & $4600 \%$ & $1150 \%$ \\
CYP2C 9.5 & $2100 \%$ & $2100 \%$ \\
\hline
\end{tabular}




\section{CHAPTER IV}

4. Summary and Conclusions 
To date, several allelic variants of CYP2C9 have been discovered in a number of diverse populations throughout the world. With the prevalence of the variants becoming better known, it is important to study their effects in order to determine how they might affect drug metabolizing capacity and, possibly, prevent adverse drug reactions from occurring in patients. It is also important to study drug interactions of these allelic forms. Recently, positive heterotropic cooperativity, or activation of substrate metabolism, has become a more frequently reported drug interaction. There are many examples of this phenomenon in the literature for CYP2C9 as well as CYP3A4. However, activation has not been studied in allelic variant forms of CYP2C9.

We have shown that dapsone activates flurbiprofen and naproxen metabolism by the CYP2C9 allelic variants to different extents. In all cases, the increase in efficiency (Vmax $/ \mathrm{Km}$ ) was greater in the variants as compared to wild type enzyme. In previous work, our lab has offered evidence of dapsone binding in the active site along with the substrate molecule causing the substrate to bind more productively. The results in this work also imply this to be the case, since the presence of dapsone increased the metabolism of flurbiprofen and naproxen for all the allelic forms studied and caused naproxen demethylation to take on a hyperbolic character, possibly by filling one of the two binding sites thought to exist in CYP2C9 for naproxen. While we have identified that the amino acids substitutions of the allelic variants seem to play a role in dapsone activation, it is still unclear what that role may be. The amino acid substitutions may alter substrate or effector binding directly, or may alter the folding of the protein changing the characteristics of the active site. However, other possibilities may exist, and there is an on-going effort to determine the nature of dapsone activation of CYP2C9 mediated 
metabolism, as well as, the consequences on the activities of the allelic variant forms of CYP2C9. 


\section{REFERENCE LIST}


Aithal GP, Day CP, Kesteven PJ, and Daly AK (1999) Association of polymorphisms in the cytochrome P450 CYP2C9 with warfarin dose requirement and risk of bleeding complications. Lancet 353:717-719.

Bhasker CR, Miners JO, Coulter S, and Birkett DJ (1997) Allelic and functional variability of cytochrome P4502C9. Pharmacogenetics 7:51-58.

Crespi CL and Miller VP (1997) The R144C change in the CYP2C9*2 allele alters interaction of the cytochrome P450 with NADPH:cytochrome P450 oxidoreductase. Pharmacogenetics 7:203-210.

Dickmann LJ, Rettie AE, Kneller MB, Kim RB, Wood AJ, Stein CM, Wilkinson GR, and Schwarz UI (2001) Identification and functional characterization of a new CYP2C9 variant (CYP2C9*5) expressed among African Americans. Mol Pharmacol 60:382-387.

Furuya H, Fernandez-Salguero P, Gregory W, Taber H, Steward A, Gonzalez FJ, and Idle JR (1995) Genetic polymorphism of CYP2C9 and its effect on warfarin maintenance dose requirement in patients undergoing anticoagulation therapy. Pharmacogenetics 5:389-392. 
Galetin A, Clarke SE, and Houston JB (2002) Quinidine and haloperidol as modifiers of CYP3A4 activity: multisite kinetic model approach. Drug Metab Dispos 30:15121522.

Gotoh O (1992) Substrate recognition sites in cytochrome P450 family 2 (CYP2) proteins inferred from comparative analyses of amino acid and coding nucleotide sequences. J Biol Chem 267:83-90.

Guengerich FP (1995) Human cytochrome P450 enzymes, in Cytochrome P450 :

Structure, Mechanism, and Biochemistry (Ortiz de Montellano PR ed) pp 473535, Plenum Press, New York.

Haining RL, Hunter AP, Veronese ME, Trager WF, and Rettie AE (1996) Allelic variants of human cytochrome P450 2C9: baculovirus-mediated expression, purification, structural characterization, substrate stereoselectivity, and prochiral selectivity of the wild-type and I359L mutant forms. Arch Biochem Biophys 333:447-458.

Hashimoto Y, Otsuki Y, Odani A, Takano M, Hattori H, Furusho K, and Iui K (1996) Effect of CYP2C polymorphisms on the pharmacokinetics of phenytoin in Japanese patients with epilepsy. Biol Pharm Bull 19:1103-1105.

Hutzler JM, Hauer MJ, and Tracy TS (2001) Dapsone activation of CYP2C9-mediated metabolism: evidence for activation of multiple substrates and a two-site model. Drug Metab Dispos 29:1029-1034. 
Hutzler JM, Kolwankar D, Hummel MA, and Tracy TS (2002) Activation of CYP2C9Mediated Metabolism by a Series of Dapsone Analogs: Kinetics and Structural Requirements. Drug Metab Dispos 30:1194-1200.

Ieiri I, Tainaka H, Morita T, Hadama A, Mamiya K, Hayashibara M, Ninomiya H, Ohmori S, Kitada M, Tashiro N, Higuchi S, and Otsubo K (2000) Catalytic activity of three variants (Ile, Leu, and Thr) at amino acid residue 359 in human CYP2C9 gene and simultaneous detection using single-strand conformation polymorphism analysis. Ther Drug Monit 22:237-244.

Imai J, Ieiri I, Mamiya K, Miyahara S, Furuumi H, Nanba E, Yamane M, Fukumaki Y, Ninomiya H, Tashiro N, Otsubo K, and Higuchi S (2000) Polymorphism of the cytochrome P450 (CYP) 2C9 gene in Japanese epileptic patients: genetic analysis of the CYP2C9 locus. Pharmacogenetics 10:85-89.

Kidd RS, Curry TB, Gallagher S, Edeki T, Blaisdell J, and Goldstein JA (2001) Identification of a null allele of CYP2C9 in an African-American exhibiting toxicity to phenytoin. Pharmacogenetics 11:803-808.

Korzekwa KR, Krishnamachary N, Shou M, Ogai A, Parise RA, Rettie AE, Gonzalez FJ, and Tracy TS (1998) Evaluation of atypical cytochrome P450 kinetics with twosubstrate models: evidence that multiple substrates can simultaneously bind to cytochrome P450 active sites. Biochemistry 37:4137-4147. 
Lee CR, Goldstein JA, and Pieper JA (2002) Cytochrome P450 2C9 polymorphisms: a comprehensive review of the in- vitro and human data. Pharmacogenetics 12:251263.

Leemann T, Transon C, and Dayer P (1993) Cytochrome P450TB (CYP2C): a major monooxygenase catalyzing diclofenac 4'-hydroxylation in human liver. Life Sci 52:29-34.

Miners JO, Coulter S, Tukey RH, Veronese ME, and Birkett DJ (1996) Cytochromes $\mathrm{P} 450,1 \mathrm{~A} 2$, and 2C9 are responsible for the human hepatic O-demethylation of Rand S-naproxen. Biochem Pharmacol 51:1003-1008.

Nakamura H, Nakasa H, Ishii I, Ariyoshi N, Igarashi T, Ohmori S, and Kitada M (2002) Effects of endogenous steroids on CYP3A4-mediated drug metabolism by human liver microsomes. Drug Metab Dispos 30:534-540.

Rettie AE, Korzekwa KR, Kunze KL, Lawrence RF, Eddy AC, Aoyama T, Gelboin HV, Gonzalez FJ, and Trager WF (1992) Hydroxylation of warfarin by human cDNAexpressed cytochrome P-450: a role for P-4502C9 in the etiology of (S)-warfarindrug interactions. Chem Res Toxicol 5:54-59.

Rettie AE, Wienkers LC, Gonzalez FJ, Trager WF, and Korzekwa KR (1994) Impaired (S)-warfarin metabolism catalysed by the R144C allelic variant of CYP2C9. Pharmacogenetics 4:39-42. 
Rodrigues AD, Kukulka MJ, Roberts EM, Ouellet D, and Rodgers TR (1996) [O-methyl 14C]naproxen O-demethylase activity in human liver microsomes: evidence for the involvement of cytochrome P4501A2 and P4502C9/10. Drug Metab Dispos 24:126-136.

Shou M, Grogan J, Mancewicz JA, Krausz KW, Gonzalez FJ, Gelboin HV, and Korzekwa KR (1994) Activation of CYP3A4: evidence for the simultaneous binding of two substrates in a cytochrome $\mathrm{P} 450$ active site. Biochemistry 33:64506455.

Steward DJ, Haining RL, Henne KR, Davis G, Rushmore TH, Trager WF, and Rettie AE (1997) Genetic association between sensitivity to warfarin and expression of CYP2C9*3. Pharmacogenetics 7:361-367.

Takanashi K, Tainaka H, Kobayashi K, Yasumori T, Hosakawa M, and Chiba K (2000) CYP2C9 Ile359 and Leu359 variants: enzyme kinetic study with seven substrates. Pharmacogenetics 10:95-104.

Tracy TS, Hutzler JM, Haining RL, Rettie AE, Hummel MA, and Dickmann LJ (2002) Polymorphic variants (CYP2C9*3 and CYP2C9*5) and the F114L active site mutation of CYP2C9: effect on atypical kinetic metabolism profiles. Drug Metab Dispos 30:385-390. 
Tracy TS, Marra C, Wrighton SA, Gonzalez FJ, and Korzekwa KR (1996) Studies of flurbiprofen 4'-hydroxylation. Additional evidence suggesting the sole involvement of cytochrome P450 2C9. Biochem Pharmacol 52:1305-1309.

Tracy TS, Marra C, Wrighton SA, Gonzalez FJ, and Korzekwa KR (1997) Involvement of multiple cytochrome $\mathrm{P} 450$ isoforms in naproxen O-demethylation. Eur J Clin Pharmacol 52:293-298.

Tracy TS, Rosenbluth BW, Wrighton SA, Gonzalez FJ, and Korzekwa KR (1995) Role of cytochrome P450 2C9 and an allelic variant in the 4'- hydroxylation of (R)- and (S)-flurbiprofen. Biochem Pharmacol 49:1269-1275.

Veronese ME, Mackenzie PI, Doecke CJ, McManus ME, Miners JO, and Birkett DJ (1991a) Tolbutamide and phenytoin hydroxylations by cDNA-expressed human liver cytochrome P4502C9. Biochem Biophys Res Commun 175:1112-1118.

Veronese ME, Mackenzie PI, Doecke CJ, McManus ME, Miners JO, and Birkett DJ (1991b) Tolbutamide and phenytoin hydroxylations by cDNA-expressed human liver cytochrome P4502C9. Biochem Biophys Res Commun 175:1112-1118.

Yamazaki H, Inoue K, Chiba K, Ozawa N, Kawai T, Suzuki Y, Goldstein JA, Guengerich FP, and Shimada T (1998) Comparative studies on the catalytic roles of cytochrome P450 2C9 and its Cys- and Leu-variants in the oxidation of 
warfarin, flurbiprofen, and diclofenac by human liver microsomes. Biochem Pharmacol 56:243-251.

Yasar U, Aklillu E, Canaparo R, Sandberg M, Sayi J, Roh HK, and Wennerholm A (2002) Analysis of CYP2C9*5 in Caucasian, Oriental and Black-African populations. Eur J Clin Pharmacol 58:555-558.

Zhao J, Leemann T, and Dayer P (1992) In vitro oxidation of oxicam NSAIDS by a human liver cytochrome P450. Life Sci 51:575-581. 